\title{
Communication Issues in Requirements Elicitation: A Content Analysis of Stakeholder Experiences
}

\author{
Jane Coughlan, Mark Lycett and Robert D. Macredie \\ Department of Information Systems and Computing \\ Brunel University \\ Uxbridge, UB8 3PH, UK \\ Email: Jane-Lisa.Coughlan@brunel.ac.uk
}

\begin{abstract}
The gathering of stakeholder requirements comprises an early, but continuous and highly critical stage in system development. This phase in development is subject to a large degree of error, influenced by key factors rooted in communication problems. This pilot study builds upon an existing theory-based categorisation of these problems through presentation of a four-dimensional framework on communication. Its structure is validated through a content analysis of interview data, from which themes emerge, that can be assigned to the dimensional categories, highlighting any problematic areas. The paper concludes with a discussion on the utilisation of the framework for requirements elicitation exercises.
\end{abstract}

Keywords: Communication; Requirements elicitation; Content analysis

\section{Introduction}

Requirements, in essence, can be said to be the embodiment of everything a user values [14]. The successful elicitation of these values, however, demands an understanding of the specific problem and its context, in which the requirements are embedded - both locally and in the wider organisation. This environment is highly complex, requiring many difficult communication interfaces to be made, particularly between the users' social/technical world and the analysts' technical world [21], forcing stakeholders involved in the requirements elicitation (RE) process to walk a tightrope between the two. Maintaining a balance, or 'fit', between the constraints of the two worlds is fraught with complexity by virtue of the behavioural nature of the process, where human and organisational elements interplay with the technical to have an impact on design $[12,1]$. Therefore, in reality, eliciting requirements involves an approach that must allow for negotiation and collaboration with all stakeholders, so that relationships can be constructed that in turn provide strong foundations for the requirements to emerge as part of a highly interactive and involved RE process. 
As a consequence, eliciting requirements involves activities that are intensely communicative and increase in significance when one considers the 'culture gap' [51] or basic semantic differences dividing two groups such as users and designers attempting to engage in meaningful dialogue [6]. There are numerous examples to be found from studies in the literature on the problems that plague projects and hinder the communication of requirements [44]. Of particular benefit, however are those studies attempting to provide insights into the real-life practice of the requirements process to complement theory [e.g., 12, 33, 19]. Indeed, the study reported in this paper is directly borne out of such a vein of research in its adaptation of a four dimensional framework used previously to analyse the communication of requirements within socially-oriented methodological approaches [10]. The communication perspective on RE put forward in the previous theoretical study, is capitalised on here, through a reassertion and confirmation of the perspective in the provision of support for the framework and its conclusions with empirical evidence drawn from the analysis of interview data. The guiding motivation of this paper is to supplement the previous work with some real-life context and commentary from individuals directly involved in the arduous task of eliciting requirements.

With this in mind, the paper is divided into five additional sections. Section 2 presents the theoretical framework, its four dimensions and associated categories along with support from the literature on the communication of requirements. Section 3 describes the research study, interviewee sample and the settings providing the context of analysis. Section 4 outlines the method for data analysis and presents the findings in the form of tabulated lists of numbered themes, organised by dimensional categories, supplemented with real-life commentary from the individuals involved. Section 5 concludes the paper, providing an overview of the findings, limitations of the study and directions for future research. Section 6 discusses the lessons that can be learnt from the study and the applicability of the framework, in terms of its benefit for organisations attempting to capture and communicate requirements.

\section{Requirements elicitation: Toward a framework for communication}

The four dimensions of the framework as presented here have been adapted from a classification scheme by Coughlan and Macredie [10]. The four key areas under consideration are: Dimension 1: Stakeholder ${ }^{1}$ participation and selection; Dimension 2: Stakeholder interaction; Dimension 3: Communication activities; and Dimension 4:

\footnotetext{
${ }^{1}$ The term 'stakeholder' replaces the term 'user' and 'user-designer' for dimensions (1) and (2) respectively, as previously employed in Coughlan and Macredie [10]. This is in order to refer to a wider range of stakeholder than just the end user.
} 
Techniques. These comprise the dedicated structure of activities performed during the requirements process as part of engaging stakeholders in the design process [23]. They have been identified as being of relevance to both practitioners and researchers alike in trying to understand and pinpoint the areas that have proved problematic in the elicitation and effective communication of requirements. The dimensions can be further broken down into categories (discussed in Sections 2.1-2.4), which can be classified on levels of: type, mediator, class and behaviour. Figure 1 below provides an illustration of the communication framework for RE and its structural components.

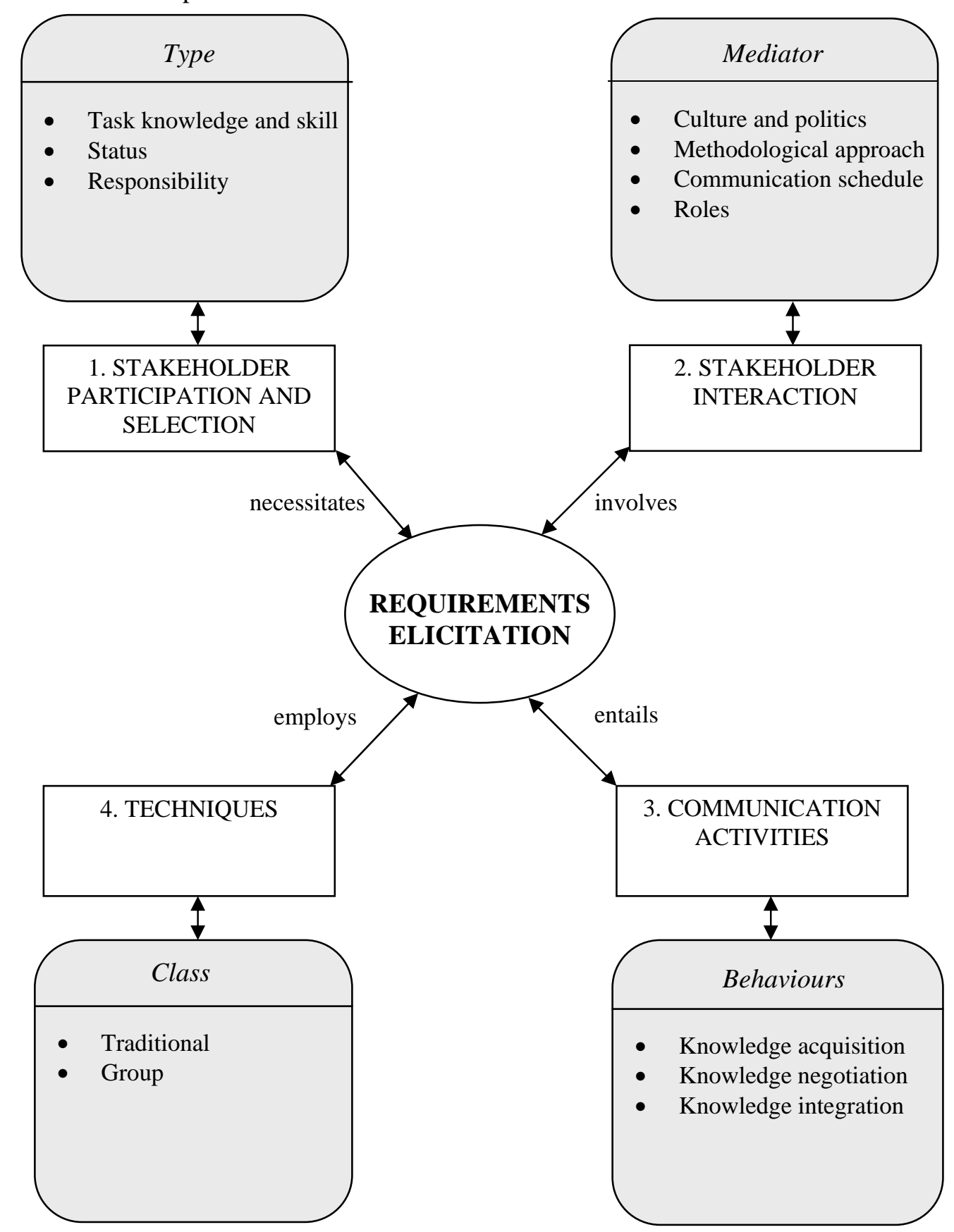

Figure 1: Four-dimensional view on requirements elicitation (adapted from Coughlan and Macredie [10]). 


\subsection{Stakeholder participation and selection}

A variety of stakeholders are involved in the execution of RE processes and the composition of the project team is all-important as it has a strong bearing on project outcomes, given that communication can be hampered by the inclusion of inappropriate people. Business consultants and project managers are two examples of types of stakeholder who are of particular interest to the RE process, as their remit requires strong communication skills [24]. Project managers govern the entire project to successful completion and have to negotiate and co-ordinate within teams as well as providing an interface to other units, which may require explanations of the scope of the project. Business consultants are responsible for facilitation in the RE process. They have the responsibility for ensuring that the requirements are fully specified and represented so as to produce detailed models of the system or process. Both types of stakeholder have to interact with a diverse range of people from both technical and non-technical domains, which is where their communication skills and experience with problematic RE processes come to the fore.

Indeed, project managers and business consultants have to accommodate and co-ordinate many different stakeholder needs and interests. Broadly speaking, different types of stakeholders can be categorised in three main ways [34, 13]:

1. Task knowledge and skill - this should include users, from frequent users to ones who might be affected by the system so as to obtain the widest spread of domain knowledge.

2. Status - this refers to managers ranging from high-low status who will share responsibility in the human sense of ensuring implementation and acceptance of the system.

3. Responsibility - this refers to responsibility in the technical sense, design and development of the system and calculating the finance available in the budget. This category is further broken down into business consultant and project manager to highlight the themes associated with their particular areas of responsibility as shown in Table 1 (Section 4.1).

Ideally, stakeholder selection should be on the basis of skills in domain knowledge, given that this is precisely the type of knowledge that will need incorporating into a system [33], though skills in IT and the RE process is also important [25]. It is more often the case, however, that stakeholders are chosen on the basis of their position and status rather than their knowledge per se [2], though project constraints of time, budget and resources will dictate ultimately the type of stakeholders involved. The selection of appropriate candidates for participation is 
critical, as stakeholders then have to engage in a pattern of interaction according to certain mediating influences, which are discussed next.

\subsection{Stakeholder interaction}

Once a project team is established, stakeholder participation requires organisation in a fashion that allows for the stakeholders to receive the support and guidance that they need for their respective roles in the requirements process and beyond. This interaction represents the process of communication and can be mediated on at least four different levels, which comprise the categories for this dimension outlined below:

1. Culture and politics - In its most basic form, organisational culture can be defined as the pattern of basic assumptions that are accepted and used by an organisation [45]. Many typologies exist which can be used to understand the culture within an organisation from anything based on two [e.g., 17], three [e.g., 46], or more popularly four different models of culture [e.g., 42]. The latter typology of Quinn and McGrath [42] is most relevant here as it based on the nature of the transactions associated with the exchange of information in four different cultures of organisations. Therefore, they distinguish between market (the rational), adhocracy (the ideological), clan (the consensual) and hierarchy (the hierarchical), which has been shown in practice to affect the communications of project managers in the projects that they undertake [18].

2. Communication schedule - Organisational life is replete with a wide range of features such as structures, in some respects influenced by the culture and politics manifest in any given organisation [41]. The category of 'communication schedule' then, is a spin-off from the category of culture and politics outlined above. In simple terms, it can been seen as the organisation's means of establishing direct communication links, in which cultural messages are conveyed, for example in the form of policy manuals, training sessions, informal discussions or formal meetings and conferences.

3. Methodological approach - Theoretically, the choice of methodological approach shows how a structure can be imposed on the way that requirements are gathered and elicited. Different types of study (for example ethnomethodological [8] and an overwhelming number of surveys [e.g., 3, 9] have shown how in reality, there is a gap between the prescription of a methodology and its use in practice. So much so, that a documented process for RE is rarely followed, or if so, only in part [20] revealing an obvious paradox between the development of methodologies and their use [26, 39]. Making a methodology 'work' seems to involve behaviour that is more ad hoc than procedural, characterised by such behaviours as improvisation, opportunism, interruption and mutual 
negotiation [e.g., 39]. These are contingencies that require strong communication in order to be managed effectively.

4. Roles - Inevitably as part of a heterogeneous project team, stakeholders will adopt roles and relationships relative to each other [11]. The adoption of roles holds implications for communication as 'mediating posts', for the knowledge transfer of requirements. The uptake of these roles provides the means through which knowledge exploration and collaboration and negotiation (in the case of conflict) can be enabled [50]. All of these activities are important in the successful communication of requirements. The multi-way relationships in project teams, however, can prove problematic given the divide in knowledge, especially between the business and IT skill bases of the organisation. Typically, the difficulties have been in the alignment of different stakeholder inputs so that both technical and social considerations are given equal weight. The style that the project manager or business consultant adopts in the RE process determines the way that information is exchanged. Such styles have been classically described as a problem finding versus a problem-solving mode of enquiry, the former approach being of strong relevance to this paper as it fosters the development of different viewpoints and thus a shared understanding of the problem and potential solutions [5, 52, 35]. Two models of interaction that can be subsumed under this problem-finding perspective and which illustrate the importance of communication are Kensing and Munk-Madsen's [28] model of knowledge integration (on different domains of discourse in design) and Beyer and Holtzblatt's [4] model of mutual learning. The common link between these two models is that successful interactions between stakeholders necessitates co-operation in order to develop a shared understanding of a design situation that is often ambiguous in nature.

\subsection{Communication activities}

The activities that are undertaken by the design team as part of the RE process have a bearing on the degree, structure and quality of communication between team members. Indeed, Hartwick and Barki [23] identify ‘communication activity' as an important dimension of user participation in the development process. For communication to occur reliably in the elicitation of requirements, there needs to be a shared understanding, which can only occur through co-operation and negotiation. The basic and most productive behaviours of a communication activity programme revolve around knowledge acquisition, sharing and integration activities [53] and the co-ordination of the efforts involved [29].

Communication activities can be categorised by behaviour in three ways (based on [53]): 
1. Knowledge acquisition - There are links that need to be made between the different stakeholders' realms of knowledge and experience and of the technological options, so as to achieve a shared understanding and common vision of a future system. It can be seen as the preparation stage for future knowledge negotiations.

2. Knowledge negotiating - Requirements need to be negotiated as part of an iterative process, which helps to define the requirements (assuming the knowledge of requirements has been satisfactorily acquired) through a sharing of multiple stakeholder perspectives.

3. Stakeholder acceptance - Acceptance of the system implies integration of stakeholder viewpoints where both parties co-operate to understand the scope of the system and the changes that impact upon the organisation.

These activities provide a structure but they cannot be considered without relation to the techniques that act as tools for communication, which are discussed next.

\subsection{Techniques}

The techniques used in the design process are of great importance at the RE stage as they provide a useful means of facilitating communication. For example, these techniques have been defined as customer-developer links [27], which allow an exchange of information. The authors further distinguish between direct and indirect links where, from a communication perspective, direct links (and an increased number) are preferable in providing face-to-face contact and a multiplicity of cues to enrich communication and thus reduce ambiguity, which is common in a requirements capture situation.

Elicitation techniques can be distinguished by class, which can be further described in six different categories [40]. Two out of the six techniques are included in the discussion (traditional and group). Prototyping and contextual techniques and associated themes were also identified from the transcripts, but have been omitted from the present analysis as they did not meet the selection criteria on themes as defined in this study (see Section 4 for further explanation of the presentation of material used for the analysis). The remaining two techniques (cognitive and model-driven) were also omitted from the analysis as their use was unmentioned by any of the respondents in this sample and so the focus resides on the following categories of techniques:

1. Traditional - for example, questionnaires, interviews, analysis of existing documentation.

2. Group - for example, brainstorming, focus groups, consensus-building workshops. 
Depending on the context under study, some of these techniques would be more effective in the elicitation of requirements than others [22]. With regards to the RE process, there is the issue of support, where stakeholders require assistance through the difficult process of negotiating requirements, and particular techniques afford such support. For instance, group session techniques are more amenable to the active encouragement and exchange of ideas, whereas traditional techniques are limited in their support and injection of any concrete meaning into the process.

\section{Research Study: Sample and Settings}

In order to investigate further the dimensions of the framework and their applicability to RE scenarios, a series of semi-structured interviews were conducted with the aid of an interview guide [e.g., 7, 49], which was used to steer the interview within the one hour time frame for each participant. A set of specific questions was prepared, designed to probe for experiences, thoughts and opinions relating to the different dimensions of the framework (see Appendix). The questions were asked in an order as long as it did not interrupt the flow of the interview, and extra questions were asked of respondents to either seek clarification or pursue appropriate avenues of relevant enquiry.

Seven participants in total comprised the sample of respondents — two Project mangers (PM) and five Business Consultants (BC). These participants were purposively sampled, which represents one accepted way of obtaining generalisability [see 47]. As explained previously in Section 2.1, the role of the PM and BC necessitates interaction with a wide range of people from both technical and non-technical domains. Their communication experiences are therefore extremely wide ranging, and so they represent exceptional participants for inclusion in this exploratory pilot study of the validity of a four-dimensional communication framework for RE. As such theory building and the satisfaction of statistical significance is downplayed in favour of an in-depth look at two types of stakeholders' experiences of communication at the RE stage, using the proposed framework as a guide. In the absence of an agreed set of recommendations for qualitative sampling, the following four factors were taken into consideration to determine sample size:

(i) The focused nature of the study (application of a framework for communication);

(ii) The clear nature of the topic area (communication in RE);

(iii) The high quality of the data (rich and experiential with nearly 200 pages of interview transcripts);

(iv) The use of shadowed data (reports on others' experiences, such as developers). 
Given these factors suggested by Morse [37], fewer participants will be needed to reach data saturation (see the study by Low et al [32] for example, which details observational descriptions of software development projects with a similar small concentration of individuals [32]).

In terms of organisational background, the participants were employed across three different UK organisations: a bank, a software development company and an investment bank. For brevity and to indicate their main line of business, the companies are identified in the text by the titles: FinCo (bank), SoftCo (software development company), InvestCo (investment bank). These latter two companies were where the only two PM's in the study were located, leaving three BC's in FinCo and two in SoftCo. The various projects undertaken by the different companies allowed for a great deal of rich variation in experiences and approaches in the elicitation of requirements. This provides points of comparison, with which to highlight any issues in common across different organisations with reference to the four dimensions. The organisational background then provides the vehicle for elicitation experiences, although the main focus in the study was on the participants themselves (as stakeholders), referred to in the text, to ensure anonymity, by their job title and place of employment only.

\section{Data analysis}

As stated in Section 3, the number of individuals participating in this study are relatively few, compared to the more typical survey based research on the RE process [e.g., 9]. Qualitative interview studies of this kind, however, make up for in depth what they lack in breadth. For example, Singer [48] conducted an interview study on software engineers in order to identify five common 'truths' across environments on software maintenance, which collectively shed light on this process and on the best type of tool use. The purpose of the present analysis similarly looked for common 'truths' on RE, but in the form of emergent themes, derived through a content analysis of the transcribed data. Content analysis was originally devised for (and has been mainly limited to) use as a quantitative tool, where the units of analysis have been analysed primarily on measures of frequency [30]. However, the on-going debate in the literature does suggest that content analysis can also be qualitative in terms of conducting a thematic analysis [e.g., 38]. Thematic analysis of this kind [e.g., 31, 16, 43] is highly appropriate to this study as the themes that are identified provide a useful 'road map' of the patterns of relationships within and between the dimensions and categories. 
The themes were generated by following a basic, but systematic, coding procedure conducted manually [see 49,15]. This took the unit of the analysis as the complete concept of a respondent's utterance, typically ranging from a few words to an entire paragraph to which codes are attached [36]. These codes act as labels on the chunks of data, which represent the theme prevalent in that section of text, which is then assigned to the predefined categories for each dimension (presented in Tables 1-4). The total number of themes coded was 73. However, 28 (38\%) of these themes were generated by one or more individuals in the same organisation and so are excluded from the analysis presented here. This is to avoid the possibility that they may be specific to that particular organisation (though of no less importance, it should be noted) but a larger sample may be required to validate their future inclusion. Therefore, 45 (62\%) of these themes are now addressed in the analysis. These have been identified by more than one organisation and so are held to be in common across at least two different organisations. The number of themes held in common across all three organisations in this sample is 11 (24\%) and are clearly identified in the discussion where appropriate.

The ensuing discussion of the themes is related firstly by way of a tabulated summary of the themes for each category by dimension, expanded on in sections 4.1.1-4.4.4. Each theme, numbered for easy reference, is then selected in turn and supported by a narrative description. This is typically verbatim extracts from the transcripts, delivered from the point of view of one of the respondents, so as to detail one clear example of the theme's occurrence in practice, as seen through the eyes of key stakeholders, namely project managers and business consultants.

\subsection{Stakeholder participation and selection}

Table 1 presents nine themes assigned to the categories of dimension 1: Stakeholder participation and selection (see Fig. 1) amounting to $20 \%$ of the total number of themes. 


\begin{tabular}{|c|c|}
\hline $\begin{array}{c}\text { CATEGORY } \\
\text { (and Section number) }\end{array}$ & THEME \\
\hline 4.1.1 Task knowledge and skill & $\begin{array}{ll}\text { 1. } & \text { Domain knowledge } \\
\text { 2. } & \text { Business/IT experience } \\
\text { 3. } & \text { Restricted access }\end{array}$ \\
\hline 4.1.2 Status & $\begin{array}{ll}\text { 1. } & \text { Decision-makers } \\
\text { 2. } & \text { Seniority }\end{array}$ \\
\hline 4.1.3 Responsibility & $\begin{array}{l}\text { BC } \\
\text { 1. Facilitation } \\
\text { 2. Communication } \\
\text { PM } \\
\text { 3. Devising stakeholder plans } \\
\text { 4. Facilitating communication }\end{array}$ \\
\hline
\end{tabular}

Table 1: Categories and themes relating to the dimension of stakeholder participation and selection

\subsubsection{Task Knowledge and skill}

Theme one, domain knowledge was one that secured agreement from all seven interviewees where the typical statement was that stakeholders need to be brought in to a project where they have specific domain knowledge to offer in a project. Theme two, business/IT experience was one that emerged in relation to stakeholder selection and appropriate representation. A BC from FinCo explained how he tried to select business stakeholders “...who knew the systems and were used to working with IT people as well”. If themes one and two can be said to be on the wish list of stakeholder attributes, theme three, restricted access shows that there are barriers to full participation, time being the main inhibitor, which reduced the availability of stakeholders. As a BC from FinCo explained, “...we will target particular people, but quite often we'll get delegates or delegates of delegates”. This theme of restricted access is expanded on elsewhere (see Section 4.2.4, category: roles; themes 2 and 3: role stretching; abuse of position).

\subsubsection{Status}

Theme one gained unanimous agreement from interviewees' on the selection of decisionmakers for participation in a project. As a BC from FinCo explained "you may aim for a manager of that area, but then they send someone else in their place who then can't make the decisions and they have to take it back to the manager...there's a gap in the circle if you like”. This gap in continuity was the main complaint of the interviewees as it disrupts communication as the process of decision making slows down. This was a problem in UK projects, but the lack of decision-makers was also a major problem in foreign projects for more cultural reasons (see Section 4.2.1, for a discussion on the influences of culture and politics). Theme two shows that status implies a level of seniority. The general opinion was that the business should be ultimately responsible for driving the project, as technological 
impacts are the greatest in this area. So you would need "...people from the business at the highest level and pushing forward...that sell the story to the rest of the business as well”, opined a BC from SoftCo. Seniority is also implicated, in terms of respect garnered for effective change management (see Section 4.3.4, category: stakeholder acceptance; theme 3: change management).

\subsubsection{Responsibility}

The main task of the $\mathrm{BC}$ is indicated by theme one, facilitation. This places a lot of responsibility on the consultant as they have to ensure the workshops run successfully and to schedule, while trying to foster discussions and understanding in all of the stakeholders present. They also have to record the requirements, which leads to theme two, responsibility for the communication of the requirements, which applies to giving feedback to the stakeholders as well as reporting back to their superiors. The project manager's responsibilities are similar in nature but broader in scope. Theme three shows that they are responsible for devising stakeholder plans, compiling the list of individuals that will sit on the project and acting as a central node for communication. Theme four shows that they are responsible for facilitating communication among all the major stakeholders and between different working groups.

\subsection{Stakeholder interaction}

Table 2 presents eighteen themes assigned to the categories of dimension 2: Stakeholder interaction (see Fig. 1). The category of culture and politics contains themes that are not applicable across organisations. Therefore, this category is removed from the calculation of dimension 2's proportion of themes, which now amounts to $18 \%$. 


\begin{tabular}{|c|c|}
\hline $\begin{array}{c}\text { CATEGORY } \\
\text { (and Section number) }\end{array}$ & THEME \\
\hline 4.2.1 Culture and politics & $\begin{array}{l}\text { InvestCo } \\
\text { 1. Motivational/consensual } \\
\text { SoftCo } \\
\text { 2. Lead-lag } \\
\text { 3. Strict procedures } \\
\text { 4. Clear demarcation of roles } \\
\text { 5. 'Blame' culture } \\
\text { 6. Rapport/relationships } \\
\text { 7. Suspicion of the IT supplier } \\
\text { FinCo } \\
\text { 8. Business and IT camps } \\
\text { 9. Highly project based } \\
\text { 10. Laid back }\end{array}$ \\
\hline 4.2.2 Communication schedule & $\begin{array}{ll}\text { 1. } & \text { No formal structure } \\
\text { 2. } & \text { Emails/posters/visits } \\
\text { 3. } & \text { Meetings } \\
\end{array}$ \\
\hline 4.2.3 Methodological approach & $\begin{array}{l}\text { 1. Named methods/framework } \\
\text { 2. Deviations }\end{array}$ \\
\hline 4.2.4 Roles & $\begin{array}{ll}\text { 1. } & \text { Clarity and understanding } \\
\text { 2. } & \text { Role stretching } \\
\text { 3. } & \text { Abuse of position }\end{array}$ \\
\hline
\end{tabular}

Table 2: Categories and themes relating to the dimension of stakeholder interaction

\subsubsection{Culture and politics}

The themes identified in this section of Table 2 represent the collective of themes pertaining to cultural and political issues within the three companies in the study, which display clear and marked differences which accounted for, and provided the backdrop to, the communication (and its success) that takes place. Theme one refers to InvestCo's culture, which was reported by the PM to be very motivational/consensual. So while the employees were described as being very able and motivated people to begin with, the fact that InvestCo's culture was one that sought consensus among people strongly encouraged communication and provided the facility for dealing with problems as and when they arose.

In contrast SoftCo dealt largely with foreign projects. Here cultural differences abounded between the SoftCo and their foreign clients and in some instances even clashed. The problematic projects were ones from Eastern Europe and Asia. Theme one, lead-lag emerged from experiences on Eastern European projects that is to say where the West led in IT concepts, the East lagged. As one of the BC's pointed out "we're maybe ten/fifteen years ahead of, you know, the rest of Eastern Europe”. Moreover, projects are “... always driven by the IT", which meant that the consultants were often only allowed to speak to members of the IT department, even on matters of business. Theme three, strict procedures, shows that Eastern European projects were intent on following documented procedures and having everything written down, which on one hand can provide a structured guide to 
communication, but conversely if followed blindly, can also constrain communication. Theme four, clear demarcation of roles shows how people's domain knowledge in the East was very "compartmentalised", compared to a wider spread of knowledge in the West. The upshot of this being that you then have to go through a lot more people in order to get "the big picture”, which dilutes and slows down communication further. One of the BC's raised theme five. He pointed out that the severe lack of decision makers within Eastern Europe, forced projects to operate under a 'blame culture', where individuals were too afraid to make decisions, as errors in judgement would be punished by the loss of their jobs.

Another part of SoftCo's work was projects in Asia, most notably Japan. Theme six shows that a major theme here was rapport/relationships. Establishing good working relations is key to successful communication, but it was clear in the Japanese project that there were opposing views on relationship building. In this case, work hours were different for the two groups. The Japanese worked extremely hard to get the job done in order to be satisfied with the working relationship, whereas the British firm operated on establishing a relationship first with a view to getting the job done. Consequently, coming into a project at opposite ends served to limit the amount of rapport as the Japanese did not make themselves readily accessible. Such differences are highlighted by theme seven, suspicion of the IT supplier, (also common in Eastern European projects) which obstructed communication. This suspicion revolved around the intentions of the IT supplier and in many cases they were seen as a threat to their jobs (see Section 4.3.3, category: stakeholder acceptance, for further discussion on this theme).

In turn again, FinCo also had their own brand of culture and politics, which affected internal communications. Theme one, business and IT camps, showed that in this organisation the business and IT divisions were most clearly marked by a divide and so gaps in communication were apparent from the offset. Theme two, highly project based, exhibits a company nature entailing a large number of meetings, many of which were consistently described as "unnecessary". Overall, the culture of the bank can be described by theme three, laid back, in the sense that time management was not very strong in this organisation and neither was the giving or receiving of feedback.

\subsubsection{Communication schedule}

In terms of a communication schedule for the three companies, theme one, no formal structure was most prevalent in showing that there was an absence of a regular forum for communication. Theme two, emails/posters/visits, were highlighted by respondents as being 
useful in maintaining communication links or informing people of upcoming events. Theme three shows that communication tended to take place mostly via meetings, or steering committees that would deal with any conflicts when they arose. All seven interviewees agreed that meeting regularly was the best way for communicating and disseminating knowledge. Meetings were deemed useful in the promotion of "team involvement and understanding” according to one of the BC's from SoftCo, as long they did not waste time and so kept people's interest. This was also important in maintaining stakeholder commitment to a project (see Section 4.3.1, category: knowledge acquisition; theme 5: commitment). In addition, a clear lack of a schedule for meetings seriously inhibited communication among project members, highlighted in the (rare) instance where managers wanted to keep teams isolated (see Section 4.2.4, category roles; theme three: abuse of position, for further discussion).

\subsubsection{Methodological approach}

One of the interview questions specifically asked if there was any particular methodological approach taken in a requirements capture exercise (see Question 6 in the Appendix). So with regards to theme one, named methods/frameworks, only the PM from InvestCo stated categorically that there was "no rigid method", whereas both SoftCo and FinCo had named methods or frameworks, which was a standard for their companies. Theme two, deviations, shows how all three of the organisations fail to follow prescribed methodology to the letter. This occurs, as a BC from SoftCo explained, because "obviously different projects will have different circumstances" and the methodology has to be tailored to fit.

\subsubsection{Roles}

Theme one, clarity and understanding, shows the common importance among interviewees of an underlying understanding of every stakeholders' roles and responsibilities within a project, so that everyone can see how all the different roles fit and come together for the "big picture". Theme two shows how the understanding of people's roles becomes critical in the avoidance of role stretching. This was a situation most aptly described by the PM from SoftCo, where under the intense pressures of project schedules and limited resources people find themselves "stretched into areas that they are not normally geared up for", where in this instance technical people such as developers were recast into the role of business analyst despite the fact that they did not "speak the language of a business consultant". The upshot of role stretching leads to "misunderstandings or wrong requirements can be documented", as found by a BC from SoftCo, where developers are unable to interact with the users in such a way that befits their normal (less direct user) pattern of interaction. 
Theme three, abuse of position, raises an issue that is more serious than role stretching. This refers to instances where a manager in key positions (e.g., development and project sponsoring) who adopts an autocratic role in management can seriously inhibit communication if they are highly obstructive or use their positions of power to achieve their own ends. In SoftCo, according to the BC's, the problem manager appeared “...to keep her link developers and her GUI team separate”, which bred "so many faults" presumably as nobody was able to communicate. In FinCo, the BC's were caught up in instances where they felt used, by high status bosses, as tools to push through with their recommendations in an attempt to "fix the results".

\subsection{Communication activities}

Table 3 presents ten themes assigned to the categories of dimension 3: Communication activities (see Fig. 1) amounting to $22 \%$ of the total number of themes.

\begin{tabular}{|c|c|}
\hline $\begin{array}{c}\text { CATEGORY } \\
\text { (and Section number) }\end{array}$ & THEME \\
\hline 4.3.1 Knowledge acquisition & $\begin{array}{ll}\text { 1. } & \text { Gaps in understanding } \\
\text { 2. } & \text { Innovative thinking } \\
\text { 3. } & \text { Redundant aspects } \\
\text { 4. } & \text { Commitment } \\
\end{array}$ \\
\hline 4.3.2 Knowledge negotiation & $\begin{array}{ll}\text { 1. } & \text { Shared perspectives } \\
\text { 2. } & \text { Information exchange } \\
\text { 3. } & \text { Familiar process techniques }\end{array}$ \\
\hline 4.3.4 Stakeholder acceptance & $\begin{array}{ll}\text { 1. } & \text { Feedback } \\
\text { 2. } & \text { Fear factor } \\
\text { 3. } & \text { Change management }\end{array}$ \\
\hline
\end{tabular}

Table 3: Categories and themes relating to the dimension of communication activities

\subsubsection{Knowledge acquisition}

All the themes that are salient in knowledge acquisition revolve around setting the stage for later negotiations. Therefore, in order for effective negotiation to take place, an adequate level of understanding needs to be reached on the part of all stakeholders. However, knowledge acquisition can be hampered if stakeholders face barriers to understanding. Theme one shows that these barriers are mostly manifest as gaps in understanding, which obstruct the acquisition of knowledge and therefore uncorrected will impair understanding. The PM from InvestCo characterised this situation by explaining "there is a gap between the user's aspiration and the level of detail required by technology to actually implement that change”. There is also understanding in the sense that it is important for the clients to understand the package that the supplier has to offer. A situation highlighted by a BC from SoftCo who indicated a need for "knowledge transfer" whereby the client can understand how 
the package works and therefore how it will benefit them in regard to the processes they go through.

Theme two, innovative thinking, illustrates the difficulties in bridging gaps in understanding in terms of allowing existing assumptions to remain unchallenged, thereby stifling creativity and the generation of new ideas. As a BC from FinCo pointed out, people are "very much constrained by how they were doing things now and couldn't let go of how they did it now". Consequently, this lack of understanding and inability to project in forward-thinking terms leads to a poor definition of requirements. Theme three shows that there can be a considerable number of redundant aspects to people's jobs, more clearly visible to an outsider. So when asked as to why they do things a certain way, the PM from InvestCo found that one employee simply “couldn't tell you”, showing that some aspects of work are deeply ingrained. Trying to derive the real requirements requires an in-depth understanding and reflection on the part of the users, and effective communication techniques on the part of the consultants and managers (see Section 4.4.2, category: group - workshop for discussion on this common technique).

Theme four, commitment or buy-in to a project, is also an important component of knowledge acquisition where a clear lack of buy-in will adversely affect project outcomes. A BC from SoftCo described a banking project where the stakeholders, in this case the end-users, were shown a video once a month, fully explaining the business benefits of the new system which increased buy-in as compared to the sending out of circulars. However, commitment will vary naturally between stakeholders, given the many different factors that can affect participation, but some level of uniformity in commitment should be achieved through keeping people informed to maintain commitment.

\subsubsection{Knowledge negotiation}

Negotiation is a type of communication activity that involves the sharing of information, in particular as indicated by theme one, shared perspectives. A shared view of alternative perspectives is required in order to negotiate successfully to the benefit of all parties. The PM from InvestCo pointed out that a clash of perspectives occurs when users and developers have a narrow-minded perspective. This occurs, for example, on the part of the developers who build the software and then believe "that they're the custodian of the software and the process" (an example of a process in this case being the booking and confirming of a trade). Such a closed perspective serves to block effective negotiation where stakeholders remain blind to other member's perspectives. Theme two, information exchange can refer to, 
according to a BC from SoftCo, a “cross-fertilisation of ideas” which is necessary to bring out the requirements. However, as he also warned, "you need to communicate the information they need to know", that is information which is made relevant to them. Theme three, familiar process techniques are used to increase understanding, which can be applied to business problems. Examples of techniques used were the process of making a cup of tea applied to a business process of depositing money in a branch, or 'games' to introduce fun aspects and keep people's interest.

\subsubsection{Stakeholder acceptance}

This category revolves around the degree of acceptance and satisfaction users feel for a new system. Theme one, feedback, refers to communications obtained from users on documents after the workshop ends, often done by email particularly when stakeholders are not local. Feedback, especially via e-mail is often not forthcoming and the PM from SoftCo found that he had to go through the document himself to correct any faults. Theme two, fear factor, predominately over loss of jobs, can inhibit participation, communication and ultimate acceptance of the system. It tends to be felt more acutely in foreign projects than domestic ones, where little has been done to communicate to users the nature of the system and the changes that will ensue. A lack of clear procedures, or a dedicated change manager for dealing with change is exposed by theme three, change management, where in UK and foreign projects alike there is generally some "resistance to change" according to a BC from SoftCo. The communication of change should ideally stem from people that have authority and that users will respect. For example, in most instances IT managers have been responsible for communicating change and in these instances communication has been unsuccessful as the manager was not able to communicate change in a way that the users could accept and understand.

\subsection{Techniques}

Table 4 presents eight themes assigned to the categories of dimension 4: Techniques (see Fig. 1) amounting to $18 \%$ of the total number of themes. 


\begin{tabular}{|l|ll|}
\hline $\begin{array}{c}\text { CATEGORY } \\
\text { (and Section number) }\end{array}$ & \multicolumn{1}{|c|}{ THEME } \\
\hline 4.4 .1 Traditional & 1. & One-on-one walk throughs \\
& 2. & One-to-one interviews \\
& 3. & User documentation \\
\hline 4.4 .2 Group (Workshop) & 1. & Breaking down barriers \\
& 2. & Preparation \\
& 3. & 'Big picture' \\
& 4. & Inappropriate people \\
& 5. & Non-attendance \\
\hline
\end{tabular}

Table 4: Categories and themes relating to the dimension of techniques

\subsubsection{Traditional}

Traditional techniques prove useful in the elicitation of requirements where theme one, oneon-one walkthroughs are commonly employed. A BC from SoftCo described it as "a good communication method" as it allows the consultant to "walk side by side with the user" and explain what to the user what they should be thinking about. Similarly, theme two, conducting one-to-one interviews is useful for "getting any detailed requirements" according to a BC from FinCo as opposed to workshops which can be "bad for detail". Theme three, user documentation and its examination is a useful technique to "finding out what the requirements were for the system they have now" according to a BC from FinCo. So this provides the knowledge for the consultant as to how the system may be enhanced which can be communicated to the stakeholders.

\subsubsection{Group (Workshop)}

The workshop method is by far the most popular in the elicitation of requirements and therefore dominates the discussion on techniques. Theme one shows that its merits lie primarily in breaking down barriers in, according to a BC from SoftCo, "pulling people out of the environment, getting them talking with other users...to keep the ideas coming”, and thereby creating a relaxed atmosphere. However, despite the workshop method's many advantages, there is a caveat in its use and effectiveness as illustrated in theme two, preparation. A clear lack of preparation on the part of the stakeholders, where they "turn up cold", according to a BC from SoftCo, shows that the reasons for the workshop have not been communicated down the levels for effective participation.

In terms of the actual workshop itself and the communication that takes place in this forum, all of the interviewees rated highly the potential for workshops as a vehicle for communication. The workshop structure is helpful in showing stakeholders, as a BC from SoftCo said, "the bigger picture". This concept and theme three of the big picture is echoed throughout all the transcripts. It is very important in terms of communication as it 
demonstrates that each stakeholder and their respective knowledge and skill, status and responsibility only represents a small part which has to assimilated into the whole through the communication of information. However, the 'big picture' can become distorted by theme four, having inappropriate people present. This is an extremely common situation given that access to the most appropriate or key stakeholders can be restricted by their time schedules for workshops or even meetings. A BC from FinCo found in her recent workshop the "people who have been sent who are like so new into the job, that they couldn't possibly tell you...everything that goes on". This affects communication, as it would severely restrict any contribution to a workshop. Even if the chosen ones do attend the workshop they are generally so stretched by time and competing responsibilities that "you may get people dropping out" according to another BC from FinCo, as illustrated by theme five, nonattendance, thereby disrupting continuity once again.

\section{Conclusions}

This study has sought to present a (previously theoretically-based) communication framework on the RE process, by identifying a number of themes associated with the frameworks' dimensional categories. This study then has served to operationalise the dimensions of the framework, evolving it to a second, more improved version, through its examination of themes found in common across BC's and PM's from different organisations. Moreover, the wide ranging nature of the themes, and the interdependencies that have been identified between some of them across categories (e.g., the theme of restricted access being linked to the themes of role stretching and abuse of position) reveal a communications perspective to be an extremely enlightening approach to RE. It forces a look at organisational issues in a holistic fashion, which entails an examination of how everything functions in an organisation in relation to everything else, for which this framework can be used as a guide (see Section 6, for a discussion on implications). It also highlights the fact that communication issues are at the crux of many problems in RE which are consistently implicated in many system development project failures.

Looking at the proportions of themes assigned to different dimensional categories, a ranking order of dimensions can be obtained to see where the number of common themes seem the weightiest, the order being: 1) Communication activities; 2) Stakeholder participation and selection; 3) Stakeholder interaction and Techniques (equal weight given). Therefore, this analysis shows for example that Dimension 3: Communication activities, appears to have a number of themes that outweigh the others and may suggest that this area requires more attention than the others when trying to improve RE. Although overall (by excluding the 
category of culture and politics), the number of themes for each dimensional category appears to be fairly evenly spread, demonstrating their equal importance in the effective communication of requirements.

\subsection{Limitations and future directions}

The generalisability of the study's findings to other stakeholder groups has been limited by the small sample used for their requirements gathering experiences. However, it is strongly anticipated that future work based around this framework will support the conclusions drawn here as despite its diminutive size, the sample did display some robust phenomena, measured as common themes across organisations. Further research in this area can only enhance the results found here and develop the structure of the framework. Future research should be particularly aimed at investigating the ways in which these dimensions (and associated categories) impact on each other so that the full potential of a communications approach to requirements elicitation can be finally realised. This can be done through an in-depth case study of one or more organisations and individuals as part of a longitudinal research program. Ideally this would also involve more observational than interview work, sitting in on workshops, where many of the decisions on requirements are decided and communicated. Alternatively, it could be achieved by shadowing a business consultant or project manager, or any other type of stakeholder involved in the day-to-day practice of RE and the real-life constraints that are placed on the effective communication of requirements.

\section{Implications of the communication framework}

To provide a clear overview of the findings with regards to the lessons that can be learned for $\mathrm{RE}$, a summary is provided below for each of the dimensions of the framework:

Dimension 1: Stakeholder participation and selection - There is a general lack of understanding of types of stakeholder, reduced availability to ideal candidates and a lack of a clear process to access and identify stakeholders. In this respect, the hands of the BC and PM seem tied, as communicating with stakeholders lies within their respective realms of responsibility, but they are unable to carry out their communicative duties to the full, given the absence of key people in the RE process. Dimension 1 really represents the first stage in the RE process but it is clear that there are a number of stumbling blocks that left unaddressed, cause difficulties for later RE activities.

Dimension 2: Stakeholder interaction - RE is governed by many factors that exist 'behind the scenes' of which the influences of culture and politics are a prime example. Such 
influences are perhaps not immediately apparent, but are clearly important in mediating, if not dictating, communication within an organisation, where differences between culture and politics between organisations can explain the varying success rates of RE exercises. In these organisations, there was also a lack of an organised communication schedule to foster contact between stakeholders and also a lack of stringency in methodological approach to requirements gathering. Roles, on the other hand, are fairly clear-cut in an organisation, though it is the understanding of them and their execution, which is again amiss. Clearly, a lack of a defined plan for stakeholder interaction will mean that stakeholders are largely left in the dark as to their involvement in the RE process. Again this will have implications for later stages of the process, particularly where they are forced to engage in communication activities without the necessary knowledge to do so effectively.

Dimension 3: Communication activities - This dimension forms a tripartite division into acquisition, negotiation and acceptance behaviours. The boundaries between these behaviours are extremely fine, but serve to show the subtle shifts in emphasis that need to be made when engaging in a communication activity. First, that knowledge must be acquired in terms of understanding of requirements and commitment to the changes that will ensue, though there was a great lack of understanding found on the part of stakeholders in this sample. Secondly, knowledge once acquired, needs to be negotiated so that perspectives can be shared and an overall understanding of the problem area reached. This can be seriously inhibited if the knowledge acquired is very weak. Thirdly, once knowledge is acquired and negotiated there is a need for acceptance, where this information is consolidated and fully accepted by all parties. This is generally achieved through the establishment of change management procedures, which are developed for the express reason of communicating change and allaying any fears. However, there was a serious lack of provision made for management of change in any of the organisations in the study and this would have lasting (negative) effects for the duration of the projects and beyond.

Dimension 4: Techniques - Themes identified here were mostly attributed to the group technique of the workshop. This type of forum in many ways provides a test of the adherence to issues in the preceding three dimensions. It is here that the dimensional impacts are brought to bear in the workshop and its success therefore largely depends on how themes in the four areas have been managed. So while the workshop was generally touted as an excellent communication technique, its effectiveness was easily limited. This was achieved through the presence of inappropriate people (relating to dimension 1) and lack of preparation 
(relating to dimensions 2 and 3), which obstruct the elicitation, and effective communication of requirements.

The utility of the communication framework then lies in the fact that it clearly spotlights the top four areas that require attention in RE. From identification of these areas, the framework could be used as a project management tool at the start of projects to assist project managers/business consultants in preparing fully for the RE exercises. It could operate like a road map to ensure that all the important areas are covered, with the designated themes serving as signposts to the important components of the dimensional categories. Alternatively, it could be used 'post-project' as an evaluation/diagnostic tool to assess its success and suggest areas for improvement. However, the framework is still in its infancy, but it is anticipated that further research as outlined in Section 5.1 will aid its future refinement, with an observed outcome of its ease in accessible of use for both researchers and practitioners alike; in the short-term for gathering requirements, but more significantly longterm, in the adoption of a more holistic and communications-oriented approach to RE. 


\section{References}

1. A. Al-Rawas, S. Easterbrook, Communication problems in requirements engineering: a field study, Proceedings of the 1st Westminster Conference on Professional Awareness in Software Engineering, London, February 1996, pp. 47-60.

2. M. Beirne, H. Ramsay, A. Panteli, Participating informally: opportunities and dilemmas in user-driven design, Behaviour and Information Technology 17 (5) (1998) 301-310.

3. V. Bellotti, Implications of current design practice for the use of HCI techniques, Proceedings of the HCI Conference on People and Computers IV, Manchester, September 1988, pp. 13-34.

4. H.R. Beyer, K. Holtzblatt, Apprenticing with the customer, Communications of the ACM 38 (5) (1995) 45-52.

5. R.J. Boland, The process and product of system design, Management Science 24 (9) (1978) 887-898.

6. R.P. Bostrum, Successful application of communication techniques to improve the systems development process, Information Management 16 (5) (1989) 279-295.

7. M. Brenner, J. Brown, D. Canter, (Eds.), The Research Interview: Uses and Approaches, Academic Press, London, 1985.

8. G. Button, W. Sharrock, Occasioned practices in the work of software engineers, in: M. Jirotka, J. Goguen, (Eds.), Requirements Engineering: Social and Technical Issues, Academic Press, London, 1994, pp. 217-240.

9. P.D. Chatzoglou, Factors affecting completion of the requirements capture stage of projects with different characteristics, Information and Software Technology 39 (9) (1997) 627-640.

10. J. Coughlan, R.D. Macredie, Effective communication in requirements elicitation: a comparison of methodologies, Requirements Engineering 7 (2) (2002) 47-60.

11. N. Cross, A. Clayburn Cross, Observations of teamwork and social processes in design, in: N. Cross, H. Christiaans, K. Dorst, (Eds.), Analysing Design Activity, John Wiley \& Sons, Chichester, 1996, pp. 291-317.

12. B. Curtis, H. Krasner, N. Iscoe, A field study of the software design process for large systems, Communications of the ACM 31 (11) (1988) 1268-1286.

13. L. Damodaran, User involvement in the system design process - a practical guide for users, Behaviour and Information Technology 15 (6) (1996) 363-377.

14. A.M. Davis, The harmony in rechoirments, IEEE Software 15 (2) (1998) 6-8. 
15. I. Dey, Qualitative Data Analysis: A User Friendly Guide for Social Scientists, Routledge, London, 1998.

16. L. Dubé, D. Robey, Software stories: Three cultural perspectives on the organizational practices of software development, Accounting Management and Information Technologies, 9 (4) (1999) 223-259.

17. F.A. Dubinskas, Modeling cultures of project management, Journal of Engineering and Technology Management 10 (1) (1993) 129-160.

18. M. Elmes, D. Wilemon, Organizational culture and project leadership effectiveness, Project Management Journal, 19 (4) (1988), 54-63.

19. K.E. Emam, N.H. Madhavji, A field study of requirements engineering practices in information systems development, Proceedings of the 2nd IEEE International Symposium on Requirements Engineering, York, March 1995, pp. 68-80.

20. B. Fitzgerald, The use of systems development methodologies in practice: a field study, Information Systems Journal, 6 (1) (1997) 3-23.

21. E.J. Garrity, Synthesizing user centered and designer centered IS development approaches using general systems theory, Information Systems Frontiers, 3 (1) (2001) 107-121.

22. J.A. Goguen, C. Linde, Techniques for requirements elicitation, Proceedings of the $1^{\text {st }}$ IEEE International Symposium on Requirements Engineering, San Diego, March 1993, pp. 152-164.

23. J. Hartwick, H. Barki, Communication as a dimension of user participation, IEEE Transactions on Professional Communication 44 (1) (2001) 21-36.

24. H.F. Hofmann, Requirements Engineering: A Situated Discovery Process, Gabler, Wiesbaden, Germany, 2000.

25. H.F. Hofmann, F. Lehner, Requirements engineering as a success factor in software projects, IEEE Software 18 (4) (2001) 58-66.

26. L.D. Introna, E.A. Whitley, Against method-ism: exploring the limits of method, Information Technology and People, 19 (1) (1997) 31-45.

27. M. Keil, E. Carmel, Customer-developer links in software development, Communications of the ACM 38 (5) (1995) 33-44.

28. F. Kensing, A. Munk-Madsen, PD: structure in the toolbox, Communications of the ACM 36 (4) (1993) 78-85.

29. R.F. Kraut, L.A. Streeter, Coordination in software development, Communications of the ACM 38 (3) (1995) 69-81.

30. K. Krippendorff, Content analysis: An Introduction to its Methodology, Sage, Beverley hills, CA, 1980. 
31. E. Longmate, P. Lynch, C. Barber, Informing the design of an online financial advice system, Proceedings of the HCI'00 Conference on People and Computers XIV, Sunderland, September 2000, pp. 103-117.

32. J. Low, J. Johnson, P. Hall, F. Hovenden, J. Rachel, H. Robinson, S. Woolgar, Read this and change the way you feel about software engineering, Information and Software Technology 38 (2) (1996) 77-87.

33. M. Lubars, C. Potts, C.H. Richter, A review of the state of the practice in requirements modelling, Proceedings of the 1st IEEE International Symposium on Requirements Engineering, San Diego, January 1993, pp. 2-14.

34. L. Macaulay, Requirements capture as a cooperative activity, Proceedings of the 1st IEEE International Symposium on Requirements Engineering, San Diego, March 1993, pp. 174-181.

35. G.M. Marakas, J.J. Elam, Semantic structuring in analyst and representation of facts in requirements analysis. Information Systems Research 9 (1) (1998) 37-63.

36. M. Miles, M. Huberman, Qualitative Data Analysis, Sage, Thousand Oaks, CA, 1994.

37. J.M. Morse, Determining sample size, Qualitative Health Research 10 (1) (2000) 3-5.

38. B. Mostyn, The content analysis of qualitative research data: a dynamic, in: M. Brenner, J. Brown, D. Canter, (Eds.), The Research Interview: Uses and Approaches, Academic Press, London, 1985, pp. 115-145.

39. J. Nandhakumar, D.E. Avison, The fiction of methodological development: a field study of information systems development, Information Technology and People 12 (2) (1999) 176-191.

40. B. Nuseibeh, S. Easterbrook, Requirements engineering: a roadmap, Proceedings of the $22^{\text {nd }}$ International Conference on Software Engineering, Limerick, June 2000, pp. 35-46.

41. A.M. Pettigrew, Conclusion: organizational climate and culture: two constructs in search of a role, in: B. Schneider (Ed.), Organizational Climate and Culture, JosseyBass, San Francisco, 1990, pp. 413-441.

42. R.E. Quinn, M.R. McGrath, The transformation of organizational cultures: a competing values perspective, in: P.J. Frost, L.F. Moore, M.R. Louis, C.C. Lundberg, J. Martin, (Eds.), Organizational Culture, Sage, Newbury Park, CA, 1985, pp. 315334.

43. S. Sahay and D. Robey, Organisational context, social interpretation, and the implementation and consequences of geographic information systems, Accounting Management and Information Technology 6 (4) (1996) 255-282. 
44. H. Saiedian, R. Dale, Requirements engineering: making the connection between the software developer and the customer, Information and Software Technology 42 (6) (2000) 419-428.

45. E.H. Schein, Organizational Culture and Leadership, Jossey-Bass, San Francisco, 1985.

46. C. Scholz, Corporate culture and strategy - the problem of strategic fit, Long Range Planning 20 (4) (1987) 78-87.

47. D. Silverman, Doing Qualitative Research: A Practical Handbook, Sage, London, 2000 .

48. J. Singer, Practices of software maintenance, International Conference on Software Maintenance, Bethesda, MD, November 1998, 139-145.

49. J.A. Smith, Semi-structured interviewing and qualitative analysis, in: J.A. Smith, R. Harré, L.V. Langenhove, (Eds.), Rethinking Methods in Psychology, Sage, London, 1995, pp. 9-26.

50. D.H. Sonnenwald, Communication roles that support collaboration during the design process, Design Studies 17 (3) (1996) 277-301.

51. A. Taylor-Cummings, Bridging the user-IS gap: a study of major systems projects, Journal of Information Technology 13 (1) (1998) 29-54.

52. N.P. Vitalari, G.W. Dickson, Problem solving for effective systems analysis: An experimental exploration, Communications of the ACM 26 (11) (1983) 948-956.

53. D.B. Walz, J.J. Elam, B. Curtis, Inside a software design team: knowledge acquisition, sharing, and integration, Communications of the ACM 36 (10) (1993) 63-77. 


\title{
Appendix: Interview questions
}

\author{
Section A: Background/General \\ 1. What is your name, title and position within the company? \\ 2. How long have you been with the company and where does your experience primarily lie? \\ 3. Can you give a brief overview of the nature of the project you are currently involved in? \\ Section B: User participation and selection \\ 4. Who were the stakeholders involved in the project and what role did you play? \\ 5. Along which criteria, if any were the stakeholders chosen to participate in the project team? \\ PROMPT: Domain knowledge? Status? Responsibility? Political influences? \\ Section C: User-designer interaction \\ 6. Is there a particular methodology that is favoured for requirements capture exercises and why? \\ PROMPT: What are their values? Are they product or human-centred in outlook? \\ 7. Was it adopted in your most recent exercise? \\ 8. How was communication between the stakeholders organised with regards to the capture of requirements? \\ PROMPT: Communication structure and nature of roles for mediation - co-operative, authoritative, \\ independent (user-led)? Is the view of design as a behavioural process? Direction of communication? \\ Any tension between sense of ownership and responsibility? \\ 9. Were there any instances in this exercise where communication broke down or was constrained? How was \\ this manifest and could you identify these failures in communication? Please try to answer both in terms of: \\ (a) The exercise itself \\ (b) The wider organisation
}

PROMPT: Problems with sourcing, articulation, conflict and misunderstanding? Politics, culture?

10. How were difficulties in communication managed?

PROMPT: Authority or consensus?

11. Were there any instances where communication was successfully channelled? How was this manifest and could you identify these strengths in communication? Please try to answer both in terms of:

(a) The exercise itself

(b) The wider organisation

PROMPT: What are the effective means of communication?

Section D: Communication activities

12. How were the requirements elicited?

PROMPT: Did process follow through stages of knowledge acquisition, negotiation and integration?

13. Did this process foster an understanding, in your opinion, of the following:

(a) User needs and assist users to understand their needs further?

(b) Business needs and wider organisational requirements?

PROMPT: Any reflection of real-world accuracy in understanding the wider context and fit of intended

system - alignment?

14. Do you think the users are generally helped or hindered during the process of communicating requirements? PROMPT: Factors which support or obstruct - appropriate roles, strong team work etc?

Section E: Techniques

15. Which techniques were used to elicit requirements and for what purpose?

PROMPT: Traditional, group, prototyping, model-driven, cognitive contextual? 